\title{
Objets de médiation de la science et objets de
} design. Le cas du projet
«Design Quantique »

\section{CAMILLE JUTANT ET JULIEN BOBROFF}

Cet article s'appuie sur l'observation et l'analyse d'un dispositif pédagogique mené par l'École nationale supérieure de création industrielle (ENSCI - Les Ateliers), à Paris, et le Laboratoire de physique des solides de l'université Paris-Sud (Orsay) entre mars et juin $2013^{1}$. Le projet "Design Quantique» a réuni, pendant quatre mois, seize élèves en design, une équipe pédagogique de designers et un chercheur en physique quantique, dans le but d'expérimenter un projet de design à partir des principes fondamentaux de la physique quantique. Par " projet de design ", on entend ici un projet guidé par plusieurs caractéristiques de l'activité de design : il correspond à un travail de conception sur la forme et la fonction d'un objet, les objets conçus pourront être fabriqués industriellement, il répond à une commande et il opère par expérimentation ${ }^{2}$.

1. Nous tenons à remercier, pour leur accueil et leur participation à ces travaux, François Azambourg, Clémentine Chambond, Véronica Rodriguez et tous les participants de l'atelier.

2. Ces caractéristiques émergent à la lecture des définitions du design qu'en donnent les historiens du design et les designers. "Selon une acception désormais internationale, on peut néanmoins parler de "design" dès lors qu'il s'agit de concevoir un objet en vue d'une production industrielle et particulièrement d'une production de série. "Design" caractérise alors la part de création qui dans la conception d'un objet [...] assure la cohérence entre les impératifs techniques de fabrication, la structure interne de l'objet, sa valeur d'utilisation et son aspect ", Raymond Guidot, Histoire du Design de 1940 à nos jours, Hazan, Paris, 2004 ; Jacquie Barral et Joël Gilles (dir.), Dessein, dessin, design, publications de l'université de Saint-Étienne, 2007 ; Panton Verner, "Qu'est-ce que le design », in Alexandra Midal (dir.), Design, L'anthologie. 1841-2007, Éditions de la Cité du design, Saint-Étienne, 2013.
Cet article propose d'explorer les enjeux associés à la médiation de la science à partir de l'observation d'une collaboration de six mois entre un chercheur en physique quantique et des designers, au sein de l'atelier "Design Quantique ", mené entre I'ENSCI - Les Ateliers et le Laboratoire de physique des solides d'Orsay. Les objets produits par les designers sont-ils des objets de médiation de la science? Quels types de relations tissent-ils avec la physique quantique ? Ces relations sont-elles spécifiques de la rencontre entre chercheurs et designers ? L'hypothèse des auteurs est que les objets produits dans le cadre de cet atelier sont étrangement similaires aux dispositifs de vulgarisation classiques de la physique quantique et en même temps fonctionnent (c'est-à-dire produisent du sens) à partir de ressorts radicalement différents. Ils déploient un espace de médiation qui interroge les modalités de la communication de la science (la démonstration, la représentation, l'analogie, l'art-science, etc.).

Mots-clés: médiation de la science, vulgarisation, collaboration, design, objets, physique quantique, infra-ordinaire 
Si les collaborations entre designers et scientifiques ne sont pas nouvelles ${ }^{3}$, cet atelier est un dispositif inédit du point de vue du domaine scientifique en jeu : c'est l'une des premières fois que designers et chercheurs collaborent à partir des principes de la physique quantique ${ }^{4}$. Cette rencontre peut même paraître paradoxale : la physique quantique est non tangible et observable à une échelle trop petite pour être manipulable hors laboratoire. Son caractère mathématique, abstrait, invisible à l'œil nu et non intuitif la rend difficile à appréhender pour un public non spécialisé. D’autres sciences comme la biologie étudient également des objets microscopiques et invisibles à l'œil nu, par exemple un virus, mais qui ont des comportements classiques et peuvent être filmés ou photographiés avec les outils adaptés. La physique quantique, au contraire, propose une description mathématique des objets difficile à retranscrire en images et qui échappe à l'intuition. Par exemple, le comportement d'un atome ne ressemble à rien de connu ; il ne constitue pas, a priori, une matière première adaptée au design.

Trois raisons ont pourtant soutenu ici le choix du design.

Une hypothèse d'abord : le fait qu'il n'y a « rien à montrer » de façon directe offre a contrario un espace de liberté créatrice pour le designer, dont l'une des spécificités est de savoir mettre en forme et en matière un concept ou une idée abstraite.

Ensuite, un constat : même si elle décrit les phénomènes à très petite échelle, la physique quantique permet in fine de comprendre la matière et ses propriétés élémentaires (solidité, couleur, chaleur, magnétisme). Elle touche donc aussi à une problématique chère aux designers.

Enfin, une histoire : les partenaires du projet « Design Quantique » avaient déjà collaboré de façon fructueuse à un autre sujet de physique fondamentale, la supraconductivité ${ }^{5}$. Cette première rencontre avait révélé certains points communs aux méthodes d'expérimentation des designers et des scientifiques qui pouvaient être mis à profit dans ce nouveau projet, en particulier la démarche visant à découvrir les propriétés d'une nouvelle matière ou d'un concept par tests, essais pratiques, erreurs, manipulation, etc.

L'objectif de l'atelier était relativement ouvert : les élèves devaient « partir » de la physique quantique afin de produire une idée, un objet, un outil ou même une image, mais leur interprétation et leur appropriation de la quantique pouvaient

3. Nous ne parlons pas ici des collaborations entre sciences de l'ingénieur et designers, mais des collaborations entre design et science fondamentale : en France, une structure comme Le laboratoire expérimente ce type de collaboration depuis 2007 ; des projets comme E.Chromi, par les designers Alexandra Ginsberg et James King, dans le domaine de la biologie synthétique, en témoignent aussi.

4. La physique quantique naît au début du $\mathrm{XX}^{\mathrm{e}}$ siècle pour expliquer certaines expériences que la physique classique ne permettait pas de comprendre, notamment le comportement de la matière et de la lumière, ainsi que les particules qui les composent (atome, électron, photon, etc.). Ses applications sont nombreuses, qui vont de l'électronique moderne qui équipe les ordinateurs et appareils mobiles aux lasers ou aux cellules photovoltaïques. Elle constitue une des pierres angulaires de la physique moderne, puisqu'elle permet de comprendre à la fois la matière et les nouvelles technologies. C'est la raison pour laquelle elle est enseignée, en France, dès la terminale aux élèves qui se destinent à des études scientifiques.

5. www.supradesign.fr et « Design and Superconducting Levitation », Leonardo, MIT Press, Vol. 47, 2014, p. 475. 
rester très libres, tout comme le choix des outils de représentations ou le choix de la finalité qu'ils donnaient à leur projet. La relation avec la physique quantique était donc un point de départ et la vulgarisation n'était pas un objectif imposé. Les élèves ont, néanmoins, débuté le projet avec une initiation à la physique quantique au cours d'une visite du Laboratoire de physique des solides d'Orsay. Il a ensuite été convenu que le responsable scientifique passerait une journée par semaine avec eux, à l'ENSCI - Les Ateliers, pour répondre à leurs questions et discuter avec eux aux côtés des designers professeurs de l'École.

L'analyse que nous présentons dans cet article a été guidée par deux questions : Les objets conçus par les élèves en design, dans le cadre du projet " Design Quantique », sont-ils des objets de médiation?

Si notre analyse permet de dire que les objets de design sont bien des objets de médiation, alors, ont-ils des qualités spécifiques?

Notre première hypothèse consiste à dire que dans le cas de la communication de la physique quantique, les objets que nous avons observés déploient effectivement un espace de médiation de la science physique. En d'autres termes, ils déploient un espace de signification, ils racontent des histoires. Ces histoires s'articulent autour de deux éléments que nous décrirons plus bas - le décalage et l'hyper-quotidien -, qui sont les deux ressorts principaux de production de signification. Notre deuxième hypothèse est que cet espace de médiation interroge au moins quatre modalités de la communication de la science : 1. le recours à la représentation des propriétés des objets scientifiques, 2 . le recours à l'analogie avec l'univers du quotidien, 3. le recours à certains dispositifs de médiation (l'ouvrage, le documentaire, la démonstration devant public), enfin 4 . le rapprochement entre art et science.

Pour conclure cette introduction, on rappellera quelle a été notre position vis-à-vis du projet. Le dialogue entre les deux auteurs de cet article s'est noué à l'occasion de l'atelier "Design Quantique ", dont l'un des auteurs, physicien, était à l'initiative, avec l'équipe pédagogique de l'ENSCI - Les Ateliers, François Azambourg et Clémentine Chambon. Le second auteur menait un projet post-doctoral à l'ENSCI - Les Ateliers sur les relations entre design et sciences. Il a participé aux séances de travail entre les designers, les étudiants et le physicien, afin de contribuer, à partir d'un point de vue en sciences sociales, à une réflexion collective sur les enjeux de ce type d'atelier.

Les résultats présentés dans le présent article sont donc issus d'un double point de vue, celui d'un chercheur en physique fondamentale animé par un questionnement sur les modalités de la communication des sciences et celui d'une chercheuse en sciences de l'information et de la communication étudiant la rencontre entre des productions de design et des situations de communication dans lesquelles elles circulent (la situation pédagogique, la situation de vulgarisation). Ces résultats sont aussi issus de l'observation participante des deux auteurs, entre les mois de janvier et de juin 2013, d'une série d'entretiens avec les élèves et les équipes pédagogiques et, surtout, d'une analyse sémiotique des productions des élèves. Chaque projet a été examiné par les deux auteurs à partir d'une grille d'analyse à deux entrées : quel est le registre de médiation privilégié par l'objet ? quel est le lien que l'objet construit avec des éléments de physique quantique rencontrés par les élèves au cours du projet? 
La première partie de cet article présente notre cadrage théorique et rappelle les acquis des études sur les dispositifs de vulgarisation de la science. La deuxième partie propose une analyse comparative, concernant la physique quantique, entre objets de médiation existants et objets produits et observés dans l'atelier « Design Quantique ». La conclusion cherchera à ouvrir des pistes de réflexion sur la spécificité de ces objets au regard de la médiation qu'ils offrent à la physique quantique.

\section{LA MÉdiATION DE LA SCIENCE AU PRISME DU DESIGN}

L'apport des études sémiotiques, dès les années 1970, a permis de considérer le design comme une activité productrice de discours spécifiques, de récits et de sens ${ }^{6}$. Dans cette perspective, il existerait deux voies du design, " celle qui configure exactement la fonction particulière d'un objet et celle qui maille les territoires, fictionnels ou non, dans lesquels il se meut ${ }^{7}$. Cette perspective permet de regarder autrement la rencontre entre design et communication de la physique quantique, non plus comme un paradoxe, ou comme la " mise en forme " d'un domaine de recherche, mais plutôt comme la production d'un faisceau de relations, ou même d'histoires, entre science et société. C'est en ce sens que les objets de design rencontrent et questionnent la problématique de la médiation, car ils viennent se loger sur le terrain des questionnements actuels sur les modalités de communication de la science.

\section{Apports des recherches sur la vulgarisation scientifique}

Les études en sciences sociales sur la vulgarisation ont montré depuis les années 1960 que le champ de la vulgarisation scientifique était infiniment plus complexe à traduire que ce que l'image d'une transmission ou traduction des savoirs scientifiques par un médiateur pour des publics ignorants pouvait laisser entendre. Et ce, malgré la prégnance d'une représentation de la fonction "instructrice » de la communication de la science ${ }^{8}$. "Il n'y a pas d'un côté un discours scientifique source, discours incompréhensible par le public moyen et de l'autre un discours second, reformulation et paraphrase du premier destiné au plus grand nombre, mais un continuum, dans lequel les scripteurs, leurs textes et leurs diverses intentions se mêlent intimement. " ${ }^{9}$ Cette évolution dans le regard porté sur l'exercice de la communication de la science est le corollaire d'une réflexion épistémologique sur les représentations de la science, soit comme espace de savoirs rationnels, soit comme champ de tensions disciplinaires, soit comme lieu de

6. Jean Baudrillard, Le système des objets : la consommation des signes, Gallimard, Paris, 1968 Gary Shank, "It's Logic in Practice, My Dear Watson: An Imaginary Memoir from Beyond the Grave", Forum: Qualitative Social Research, 2(1), Art. 9, 2001; Webb Keane, "Semiotics and the social analysis of material things", Language \& Communication, 23 3-4), 2003, p. 409-425: Klaus Krippendorff, The Semantic Turn: A New Foundation for Design, CRC Press, Boca Raton, 2005.

7. Éric Vandecasteele, « les fictions du design (nains de jardin et pensée topologique) », in Jacquie Barral et Joël Gilles (dir.), Dessein, dessin, design, op. cit., p. 60.

8. Michel Callon, «Plaidoyer en faveur du réchauffement des relations entre science et société. De l'importance des groupes concernés ", L'Actualité chimique, 280-281, 2004, p. 21.

9. Daniel Jacobi, «Sémiotique du discours de vulgarisation scientifique », Semen [En ligne], 2 | 1985, mis en ligne le 21 août 2007, consulté le 30 septembre 2013. URL : http://semen.revues.org/4291. 
pratiques sociales, soit encore comme lieu d'inscription et de circulation des énoncés scientifiques ${ }^{10}$.

Par ailleurs, les études ont montré que les publics de la vulgarisation, ou les médiateurs, ne sont pas toujours ceux que l'on croit, ou tout au moins ne recouvrent pas les représentations qui sont faites à leur sujet. Suzanne de Cheveigné montre par exemple que le(s) public(s) à plus fort capital scolaire sont ceux qui manifestent la plus forte défiance vis-à-vis du discours de vulgarisation ${ }^{11}$. Plus généralement, les analyses critiques envers des dispositifs de médiation de la science jugés trop " spectaculaires » ${ }^{12}$ ainsi que les enquêtes réalisées dans les musées de sciences $^{13}$ ou encore les débats autour de « la science citoyenne » ou « la science participative ${ }^{14}$ témoignent d'attentes très fortes de la part des publics sur la relation entre science et société. En ce qui concerne les médiateurs, Dominique Cartellier ${ }^{15}$, Sandrine Reboul ${ }^{16}$ ou encore Jean-Marc Lévy-Leblond ${ }^{17}$ ont montré que les acteurs concernés par l'énonciation de la vulgarisation viennent d'horizons parfois très différents (journalistes, éditeurs, chercheurs, enseignants, médiateurs de CCSTI, animateurs sociaux, communicants, etc.).

Enfin, comme le souligne Yves Jeanneret, la vulgarisation joue des rôles multiples et se présente "comme une pratique désorientée ", soumise à des déterminations scientifiques, culturelles, politiques, pédagogiques, commerciales et sociales ${ }^{18}$. En témoignent les réflexions terminologiques sur l'abandon du mot de vulgarisation, au profit de celui de médiation, de publicisation, ou encore de communication scientifique, mais aussi la prise en compte de la vulgarisation

10. Michel Callon, "Quatre modèles pour décrire la dynamique de la science », in Madeleine Akrich, Michel Callon et Bruno Latour (dir.), Sociologie de la traduction. Textes fondateurs, Mines Paris, 2006, p. 201-251.

11. Suzanne de Cheveigné, "Enquête du CNRS sur les attentes du public vis-à-vis de la science ", CNRS Info, 381, 2000, citée par Christophe Bonneuil, « Les transformations des rapports entre sciences et société en France depuis la Seconde Guerre mondiale : un essai de synthèse », colloque Science, médias et société, 15-17 juin 2004, Lyon ENS-LSH, p. 24.

12. Martin W. Bauer, «Changement de paradigme de la communication scientifique. Un public critique pour la science commercialisée ?», Questions de communication, 21, 2012, p. 123-144.

13. Joëlle Le Marec (dir.), "Évolution des rapports entre sciences et société au musée », Culture et Musées, 10, 2007.

14. Virginie Albe, Enseigner des controverses, Presses universitaires de Rennes, 2009 ; Dominique Pestre (dir), "Sciences, Technologies, Savoirs en sociétés ", Science \& devenir de l’homme, 57/58, numéro spécial, Éditions du Seuil, 2008.

15. Dominique Cartellier, «La vulgarisation scientifique à l'heure de libre accessibilité des savoirs. Quelle place pour les médiateurs? », in Marie-Pier Luneau et Josée Vincent (dir.), La lecture : agents, pratiques, usages et discours, Volume 1, numéro 2, Groupe de recherche et d'étude sur le livre au Québec, 2010.

16. Sandrine Reboul-Touré, "Le transgénique et le citoyen dans la presse écrite ", Les Carnets du Cediscor [En ligne], 6 | 2000, mis en ligne le 30 novembre 2009, consulté le 03 août 2013. URL : http://cediscor.revues.org/342.

17. Jean-Marc Lévy-Leblond, "Science, culture et public. Faux problèmes et vraies questions ", Quaderni, 46, 2001-2002, p. 395-401.

18. Yves Jeanneret, Écrire la science. Formes et enjeux de la vulgarisation, PUF, Paris, 1994. 
comme production d'un discours et activité d'interprétation spécifiques ${ }^{19}$. Pour Daniel Jacobi, les marques linguistiques qui caractérisent cette spécificité sont de trois ordres : l'usage des figures de rhétorique, comme l'analogie et la comparaison (prépondérance de la métaphore, du paradoxe, de l'hyperbole), l'usage d'un niveau de discours métalinguistique qui explique ce qui est en train d'être dit et enfin l'usage de la paraphrase et des substitutions synonymiques vis-à-vis des termes techniques.

\section{La notion de médiation}

Dans cet article, nous avons fait le choix de retenir le mot de médiation de la science pour deux raisons.

D'une part, la notion de médiation fait l'objet d'un corpus théorique, en sciences de l'information et de la communication, sur lequel nous nous appuyons, en tant que modèle communicationnel qui se distingue de l'approche technique, pour laquelle la communication est une transmission d'un émetteur à un récepteur, autant que de l'approche interactionniste, qui conçoit la communication comme un dialogue en face-à-face ${ }^{20}$. La notion de médiation engage un modèle de la communication qui met au jour l'activité de circulation des messages et des représentations entre les différents pôles de la situation de communication, l'activité d'interprétation toujours à l'œuvre et toujours changeante et son corollaire, l'activité de création de sens.

Parler de médiation permet d'autre part de porter l'attention sur l'opérateur de médiation, c'est-à-dire le tiers extérieur aux éléments à mettre en communication, dans notre cas ces deux éléments sont la science et le(s) public(s). Ainsi, l'exposition, le livre, la démonstration sont des opérateurs, autrement dit, des objets de médiation qui ont chacun leur histoire culturelle, leur fonctionnement technique et leur "opérativité sociale ${ }^{21}$. On entend ici par « objet» toute forme produite, qu'elle soit en trois dimensions, audiovisuelle ou numérique, qu'il s'agisse d'un dispositif ou d'un texte. Un objet de médiation repose sur une médiatisation, que nous qualifierons de «mise en forme propre à un média ${ }^{22}$, et une médiation, " processus de création de sens qui se réalise grâce à la relation

19. Beaudoin Jurdant, Les problèmes théoriques de la vulgarisation scientifique, Archives contemporaines, Paris, 2009 ; Yves Jeanneret, Penser la trivialité. Vol. 1 : La vie triviale des être culturels, Lavoisier, Paris, 2008 ; Daniel Jacobi, La communication scientifique : discours, figures et modèles, Presses universitaires de Grenoble, 1999 ; Bernard Schiele, Le musée de sciences : montée du modèle communicationnel et recomposition du champ muséal, L’Harmattan, Paris, 2001 ; Marie-Françoise Mortureux, " Paraphrase et métalangage dans le dialogue de vulgarisation », Langue française, 53, 1982 p. 48-61 ; Daniel Jacobi, Bernard Schiele (éd.), Vulgariser la science, Éditions Champ Vallon, Ceyzérieu, 1988.

20. Jean Caune, Pour une éthique de la médiation : Le sens des pratiques culturelles, Presses universitaires de Grenoble, 1999 ; Marie-Christine Bordeaux, "Une médiation paradoxale : "La danse, une histoire à ma façon" ", MEI - Médiation et information, 19, 2004, p. 97-107. Jean Davallon, « Réflexions sur la notion de médiation muséale », L'Art contemporain et son exposition (1), L'Harmattan, Paris, 2002, p. 41-61.

21. Louis Quéré, Miroirs équivoques, Aubier, Paris, 1982.

22. Daniel Peraya, «Un regard critique sur les concepts de médiatisation et médiation. Nouvelles pratiques, nouvelle modélisation », Les Enjeux de l'information et de la communication, 2008. 
tissée avec un tiers par un dispositif de communication ${ }^{23}$. L'analyse du statut de l'objet et l'analyse de la démarche communicationnelle à l'œuvre sont donc indissociables.

\section{LES OBJETS DE DESIGN FACE À LA MÉDIATION DE LA PHYSIQUE QUANTIOUE}

Nous avons analysé les objets produits au cours de l'atelier «Design Quantique » en distinguant la relation que l'objet nouait avec la physique quantique, ou plus précisément, en distinguant ce que l'objet «travaillait » de la quantique. Il est apparu qu'un certain nombre de projets proposaient des transpositions des représentations mathématiques ou des comportements des objets quantiques. Néanmoins, tous les projets ne proposaient pas de transpositions et certains d'entre eux offraient bien plutôt une interprétation plastique (la dimension esthétique de la physique) ou réflexive (le rôle du créateur interprétant des éléments de physique) de la physique quantique. Il nous a donc semblé plus intéressant de chercher à comprendre ce que ces objets nous disaient, non pas tant de la physique, que des formes de vulgarisation de la physique et du travail de médiation en lui-même. C'est la dimension communicationnelle du design, dont nous avons fait l'hypothèse, que nous étudions ici, c'est-à-dire la capacité de cette activité de création à explorer les formes de circulation de la science.

L'analyse que nous avons faite des objets produits dans le cadre de l'atelier nous conduit à proposer quatre familles d'objets. La première regroupe les projets qui exploitent et questionnent la représentation des objets scientifiques comme ressort de la compréhension ; la seconde regroupe les projets qui examinent la construction d'analogies entre la physique quantique et l'univers du quotidien ; la troisième regroupe les objets qui analysent les dispositifs de médiation que sont le livre, le documentaire, la démonstration devant public ; enfin, la dernière correspond aux projets qui se situent sur le terrain des relations entre art et science.

Pour chacune des familles, nous avons choisi de présenter dans le détail un projet représentatif et de décrire très brièvement les autres projets de l'atelier.

\section{La représentation des propriétés des objets quantiques}

"Objet quantique », le projet de Paul, est un dispositif vidéo qui montre un homme manipulant une forme animée aux bords flous ${ }^{24}$. Cet objet représente la particule quantique. Placé dans un décor épuré blanc, il joue avec cette forme et chacun de ces jeux montre une propriété de l'objet quantique (l'effet tunnel, la dualité onde-particule, l'intrication, etc.). L'objet quantique est signifié de plusieurs façons : le floutage et le léger mouvement des formes rouges rendent compte de l'aspect onde de l'objet quantique. La netteté et l'immobilité de la balle orange rendent compte de l'aspect particule. Enfin, le son en continu représente l'onde, quand le bruit soudain représente la particule. Le fait de choisir un décor épuré blanc n'est pas neutre : le white cube représente une sorte d'idéal d'un espace

23. Jean Davallon, Émilie Flon, «Le média exposition », Culture et Musées, 2013, p. 19-45.

24. Le site internet www.designquantique.fr, réalisé pour l'occasion, présente le projet, ainsi que toutes les productions des élèves. La vidéo de Paul est disponible sur le site www.designquantique.fr. 
neutre et vierge, dans le sens où il serait déshistoricisé, comme s'il s'agissait d'un présent absolu. En produisant cet espace, Paul fait acte de création, mettant à distance les conditions réelles d'expérimentation des objets quantiques, dans les laboratoires des physiciens, et produit le fantasme d'un espace blanc de visibilité et de lisibilité.

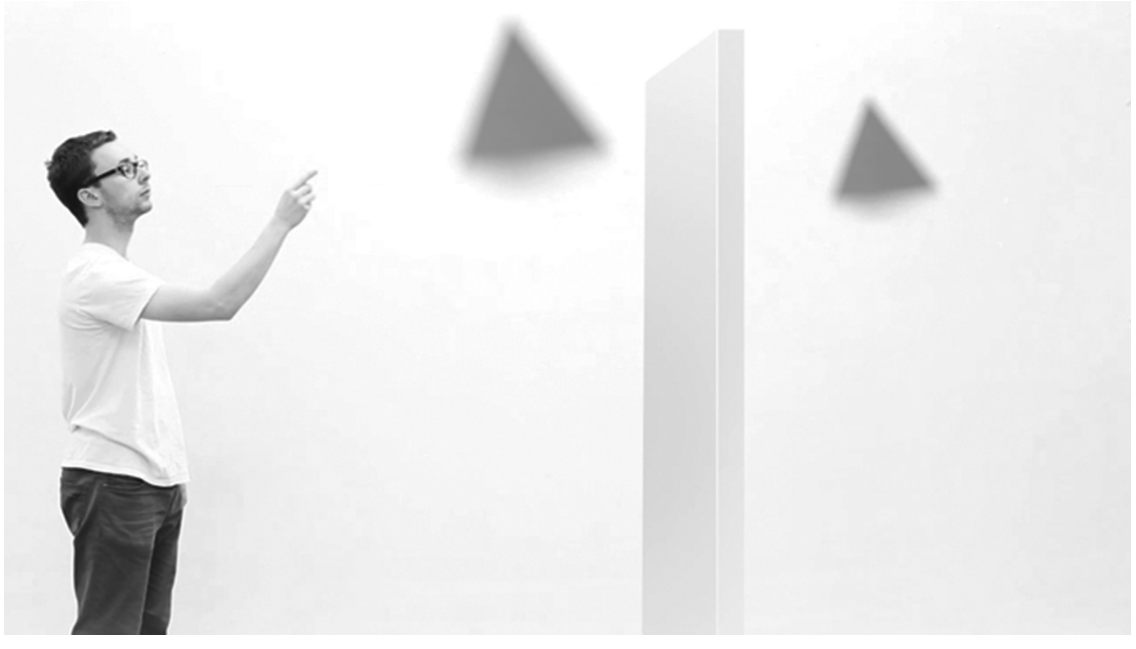

Figure 1 : Image extraite de la vidéo « Objet quantique », Paul Morin.

D’un point de vue narratif, la vidéo propose une transposition des phénomènes microscopiques et invisibles à notre propre échelle pour les rendre plus " palpables ». La présence de Paul, son bras, le haut de son corps, sa main, et le regard porté sur l'objet ramènent les objets à une échelle humaine. L'animation vidéo offre surtout une cohérence narrative et graphique, qui a pour effet de créer une logique plastique qui serait commune à chacun des phénomènes quantiques. En faisant cela, Paul propose une poétique du comportement de l'objet quantique.

Si nous comparons cet objet à une tentative analogue de représenter des effets quantiques de façon animée (les animations graphiques en trois dimensions du site Web toutestquantique.fr, par exemple), on remarque que dans les deux cas, les objets qui représentent les propriétés quantiques sont similaires (objets abstraits qui s'animent au même rythme). Le décor est pareillement épuré, bien que dans le cas des animations de vulgarisation, des règles graduées, des symboles mathématiques et des explications écrites sont présents alors que dans le cas de Paul, aucun élément graphique ni textuel, en dehors du nom de la propriété scientifique, ne donne du sens aux éléments du décor. En déshabillant la matière textuelle et scientifique et en gardant uniquement la nudité de l'objet accompagnée d'un titre qui peut sembler énigmatique, la vidéo crée un sentiment de curiosité et d'émerveillement de voir un homme manipuler cet objet comme un jeu et obtenir des effets extraordinaires. Il est à noter, donc, que Paul produit l'inverse de ce que peut produire la paraphrase dans la vulgarisation, il conserve les noms 
des propriétés sans les reformuler ou les expliciter. Il laisse opérer l'étrangeté d'expressions inconnues.

Deux autres projets interrogent également la représentation de l'objet quantique : "Pong quantique » est un jeu vidéo inspiré, dans son fonctionnement et dans sa charte graphique, du jeu Pong, développé en 1972. Les épreuves et le comportement des raquettes sont néanmoins inspirés des phénomènes quantiques (la balle est une onde puis une particule, etc.). «Quantique FM » est un dispositif sonore qui reproduit des sons comme s'ils étaient affectés par une propriété quantique (le son devient " ondulant " lorsqu'on lui applique l'effet " onde »; il se discrétise en paliers sonores lorsqu'on lui applique l'effet « quantification »).

\section{L'analogie entre l'objet quantique et l'univers du quotidien}

Le projet de Natacha est une vidéo nommée «L'appartement quantique ${ }^{25}$. Une succession de très courtes saynètes dans un appartement illustrent des analogies avec des effets de physique quantique. Parmi les figures d'analogie sémantique et sémiotique, la comparaison, la métaphore et l'analogie stricte peuvent être distinguées. Dans l'analogie stricte, c'est la relation de ressemblance structurelle entre le comparé et le comparant qui est à l'œuvre ${ }^{26}$. Dans la comparaison, ce sont les ressemblances et les différences qui sont également évaluées selon une « procédure permettant la mise en relation d'un terme A (le comparé) et de toute autre expression B (le comparant) ${ }^{27}$. Dans les deux cas, la mise en relation est faite à partir de liens explicites, à la différence de la métaphore qui « se substitue au comparé du domaine scientifique, est caractérisée par l'absence de connecteur et introduit une comparaison implicite entre le terme métaphorique et le terme scientifique auquel il se substitue ${ }^{28}$. La métaphore, plus que l'analogie et la comparaison, implique la coopération interprétative de l'auditoire.

Le projet exploite précisément la figure de la métaphore. Les objets choisis par Natacha sont des archétypes de l'univers domestique (une poêle, un pot de fleurs, un grille-pain). Chacun d'eux est mis en scène dans son espace d'utilisation habituel. Chaque plan-séquence est très court (cinq à dix secondes), sans mouvement ni montage. Des personnages désincarnés, sans visage ou de dos, apparaissent de temps en temps pour agir sur les objets (appuyer sur un bouton ou verser de l'eau). Les titres des phénomènes quantiques que chaque objet est censé représenter séparent les séquences. Ils annoncent ce que l'on va voir, mais ne disent rien des phénomènes. Exactement comme dans le cas de Paul, Natacha renonce à la paraphrase et exploite l'étrangeté d'expressions inconnues.

25. La vidéo est disponible sur le site www.designquantique.fr.

26. Néanmoins, à la différence d'une ressemblance qui serait immédiatement perceptible entre deux objets distincts appartenant au même domaine, l'analogie n'est pas le fruit d'une perception et construit de la ressemblance entre deux objets distincts appartenant à des domaines différents.

27. Daniel Jacobi, La communication scientifique, op. cit., 1999, p. 83.

28. Isabelle Collombat, «Le discours imagé en vulgarisation scientifique : étude comparée du français et de l'anglais ", metaphorik.de, 05/2003, p. 36-61. La métaphore est définie comme l'emploi d'un «mot concret pour exprimer une notion abstraite, en l'absence de tout élément introduisant formellement une comparaison » (Dubois, Dictionnaire de Linguistique, Larousse, Paris, 1973, p. 317-318)

communication \& langages $-n^{\circ} 183-$ Mars 2015 


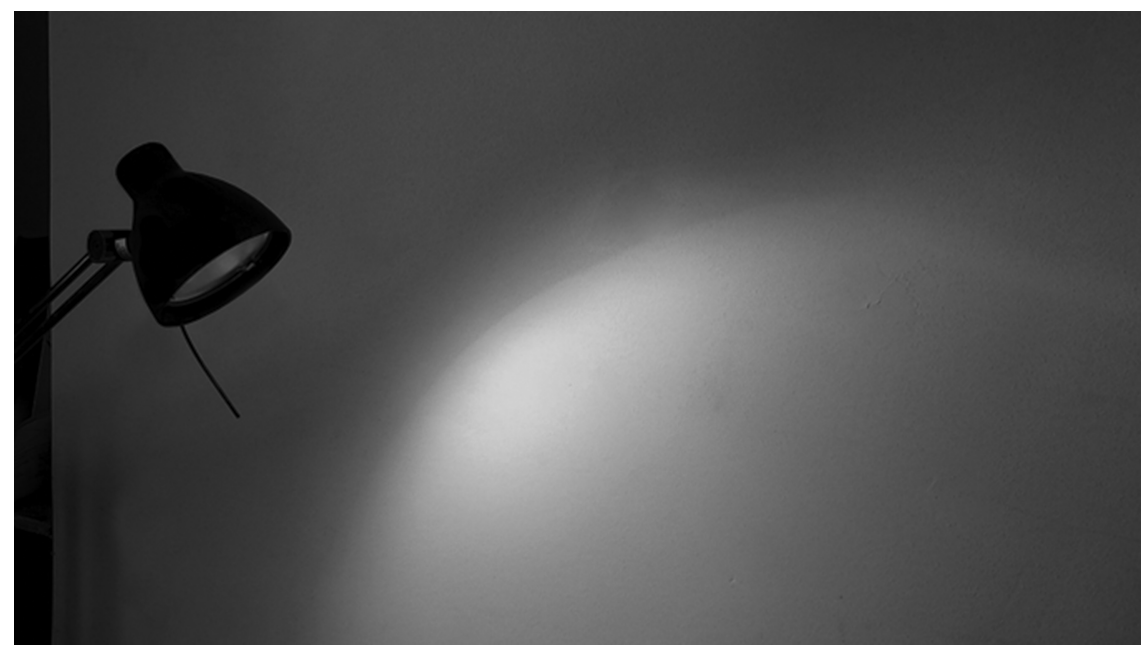

Figure 2 : Image extraite de la vidéo " L'appartement quantique ", Natacha Poutoux.

Le silence omniprésent dans la vidéo produit une tension et est un geste créatif. Il joue comme le blanc dans le cas de Paul, il produit une sorte de climat originel. Il est efficace, aussi, pour sa capacité à mettre en évidence les moindres bruits produits par l'objet quotidien en usage et concentre ainsi l'attention sur l'objet. Dans tous les cas, Natacha produit des effets spéciaux grâce à un jeu de montage relativement simple (une crêpe traverse un mur, d'un plan à un autre, par exemple). En outre, elle n'invente pas de nouveaux objets. Elle s'appuie sur des objets quotidiens et banals qui deviennent les supports de gestes tout aussi banals mais qui, mis bout à bout, produisent un sentiment d'étrangeté. La transposition d'un effet quantique en un objet ou une situation du quotidien exacerbe la singularité du phénomène quantique. En ce sens, le procédé employé par Natacha est clairement métaphorique : l'univers quotidien, en tant que comparant qui se substitue complètement au comparé, révèle l'étrangeté de l'élément comparé.

Si nous opposons ce projet au même procédé utilisé par des scientifiques pour faire comprendre l'étrangeté du monde quantique (par exemple Sven Ortoli et J. P. Pharabod qui, dans Le quantique des quantiques, livre de vulgarisation sur la quantique, proposent une analogie entre un électron et un poisson soluble), nous constatons que la transposition en référence avec notre univers quotidien ou avec des objets ou situations que nous pouvons éprouver n'est pas le privilège du projet de design. En revanche, l'unité de lieu (l'appartement), le choix d'objets et de situations que nous pourrions qualifier d'hyper-quotidiens (à l'inverse de situations plus exotiques comme pour les poissons) et surtout le séquencement du film, sans texte ni explication, ont pour effet de produire un sentiment comique d'absurde. En d'autres termes, la rencontre entre un effet quantique extra-ordinaire et un terrain de jeu infra-ordinaire, pour reprendre les termes de George Perec ${ }^{29}$, familiarise avec l'objet quantique.

29. George Perec, L’infra-ordinaire, Éditions du Seuil, Paris, 1989. 
Parmi les autres projets de l'atelier, la vidéo « Les intriqués de la vie » propose également une transposition, dans un univers quotidien, des principes de la physique quantique. Le projet «Découvrir » est un ouvrage qui propose, pour trois phénomènes quantiques, des comparaisons avec des figures de style de la rhétorique, des images d'objets quotidiens ainsi que des textes poétiques. Enfin, certains étudiants ont produit des objets s'inspirant certes de comportements quantiques, mais s'assumant comme seuls objets de design : un miroir, un luminaire ou une boîte à musique dont le comportement est modifié par la présence de l'utilisateur, ou encore des lunettes qui déforment la vision pour la rendre floue et multiple. Ces productions de design relèvent elles aussi de l'univers domestique et relèvent également du procédé de métaphore. Au-delà, elles ouvrent une question nouvelle, hors du champ de notre étude : ces objets dont l'inspiration puise dans la science fondamentale sont-ils spécifiques comparativement aux objets de design habituels, et si oui, comment s'en différencient-ils?

\section{Les dispositifs de médiation de la physique quantique}

Le projet de Marianne, "Tutti Quanta », est une démonstration, devant public, au cours de laquelle un(e) médiateur utilise une palette d'outils et de matériaux de facture simple (feuilles cartonnées, cubes en plastiques, miroirs, encre, huile, bulles) pour montrer des effets visuels qui illustrent les représentations formelles de différentes propriétés quantiques, notamment les formes géométriques des atomes (orbitales atomiques) ${ }^{30}$. Ces formes apparaissent, disparaissent, évoluent brusquement ou sont au contraire floues et ondulantes, se référant ainsi aux caractéristiques quantiques des atomes. Tous les outils et objets sont déployés à partir d'une boîte qui évoque un petit théâtre ou un cabinet de curiosités portatif. Marianne revisite le rapport entre le vulgarisateur et sa matière. Le médiateur qu'elle met en scène joue le rôle d'un marionnettiste.

D’un point de vue narratif, Marianne ne réitère aucun récit scientifique, elle présente des petits dispositifs qui produisent des formes. Elle ne dissimule pas sa façon de faire au spectateur. Elle donne, au contraire, à voir son dispositif, comme en témoigne l'importance de la présence du médiateur, qui a pour effet de concentrer l'attention sur le geste de ce dernier ainsi que sur l'opération qu'il fait subir à l'objet. Comme dans le cas de Paul, le décor est neutralisé, le théâtre des objets est l'espace d'expérimentation, et se suffit à lui-même. Marianne produit ici une fabrique de formes, une poïétique quantique.

$\mathrm{Si}$ nous comparons son projet à une animation scientifique « classique " (démonstration dans un musée des sciences ou lors d'une fête des sciences), on observe bien que dans les deux cas, le rapport à l'espace-temps de l'expérimentation est le même : le médiateur utilise un dispositif qui tient sur une petite table et donne à voir, en direct, des effets devant un public restreint. Dans les deux cas, l'effet spectaculaire de la démonstration peut susciter un émerveillement. À la différence d'un médiateur classique qui explique les phénomènes et maintient cette dimension métalinguistique du discours de vulgarisation (expliquer la démonstration, comment il la fait, pourquoi il la fait), le

30. Une vidéo de ce projet est disponible sur le site www.designquantique.fr. 


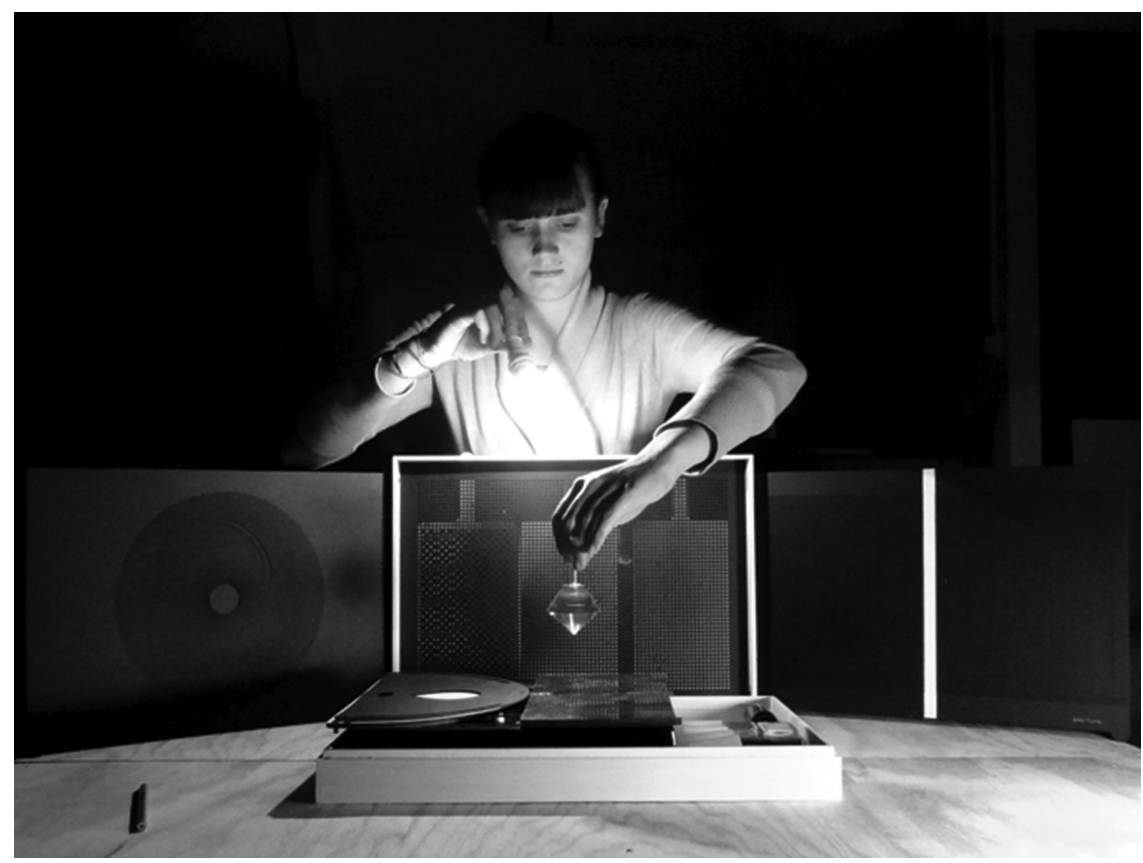

Figure 3 : Image extraite de la démonstration « Tutti Quanta ", Marianne Cardon.

présentateur de Marianne est quasiment muet. Toute l'attention du spectateur est portée sur les effets esthétiques des expérimentations, et non pas sur les effets de démonstration du discours. À ce titre, aussi, le spectacle donné à voir ne suscite pas l'interaction ou le dialogue avec le public. Il est à noter que chaque expérimentation proposée par Marianne propose une transposition rigoureuse de la représentation mathématique d'objets quantiques.

Comme le projet de Marianne, deux autres projets examinent les dispositifs de communication de la science : l'ouvrage scientifique et le documentaire scientifique. "Introduction à VI principes fondamentaux de la physique quantique » est un ouvrage, accompagné d'une série de cartes postales, d'affiches et d'un feuillet imprimé, qui propose des mises en scène des six grands principes de la physique quantique, accompagnées d'un texte explicatif. Les "Cristaux de Naïca » est une vidéo qui produit, quant à elle, une feintise, mimant un documentaire scientifique et inventant un lien entre une grotte colombienne et un principe de physique quantique.

\section{L'interprétation poétique et plastique de la physique quantique}

Le projet que nous décrivons maintenant est celui d'Émile, "Démarche sous influence ${ }^{31}$. Émile a produit un ensemble d'objets-dispositifs, qu'il a filmés. Il explique que leur fabrication est inspirée par la quantique : papiers peints, lumières

31. Une vidéo de ce projet est disponible sur le site www.designquantique.fr. 
animées, livres pour enfants, vêtements, matières, contenants, sacs à mains... À la différence de Natacha, qui utilise des objets existants, les objets présentés sont conçus pour l'occasion, mais à la différence de Marianne, ils sont des objets du quotidien. L'inspiration est soit associée à une propriété quantique, par exemple la dualité pour les vêtements à la fois liquides et solides, soit associée pour la méthodologie et le processus créatif au comportement quantique (incertitude de la mesure, saut brutal, plusieurs possibilités en parallèle...) : Émile explore la physique quantique pour influencer son propre comportement créatif et sa méthodologie de travail.

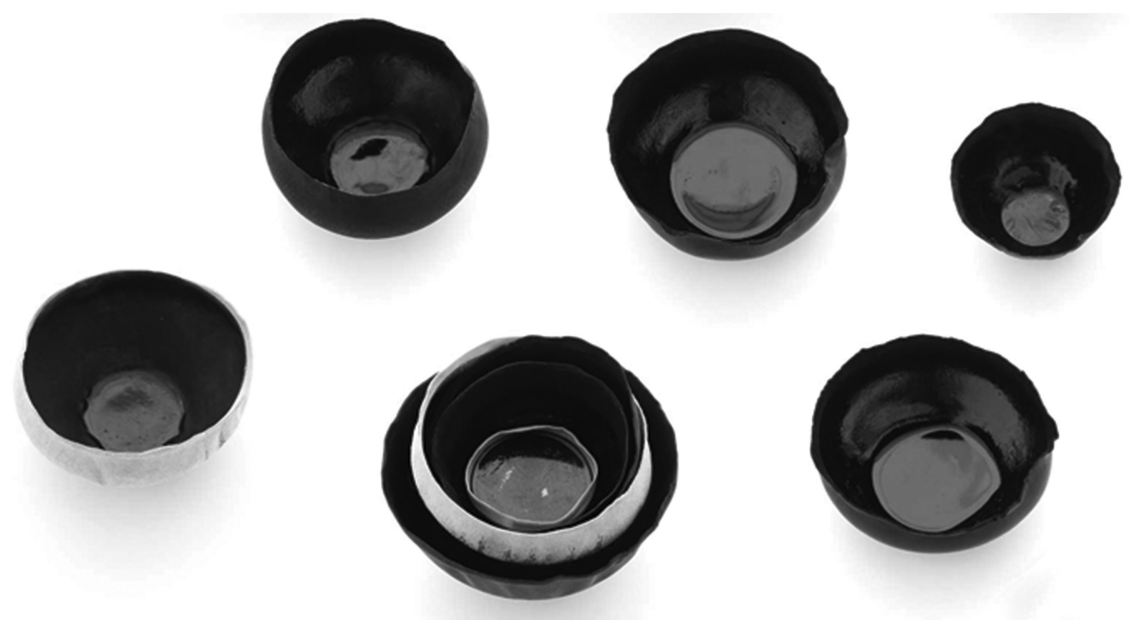

Figure 4 : Image extraite de l'exposition des objets « Démarche sous influence ", Émile Kirsch.

Le rapport entre l'objet et le propos scientifique n'est pas rigoureux. Il s'inspire délibérément d'un contresens. Le vêtement liquide et solide à la fois, comme interprétation de la superposition d'états qui caractérise l'électron, n'a plus rien à voir avec la quantique, mais avec un autre champ scientifique, la thermodynamique. La science est ici un prétexte pour construire un discours. Pas de paraphrase, pas de dimension métalinguistique du discours, pas d'analogies visant à comprendre un propos scientifique, mais au contraire production de signes et d'une posture réflexive.

Dans le domaine de la quantique, on peut comparer ce projet avec un objet relevant du champ art-science, par exemple le travail artistique de Julian Voss Andreae «Quantum objects » consistant à produire des sculptures, parfois monumentales, inspirées de la quantique, d'un point de vue plastique. Dans les deux cas, on constate un même abandon de toute volonté pédagogique ou démonstrative. Néanmoins, dans le cas d'Émile, la variété des supports et des matériaux explorés, la production d'objets quotidiens et utiles, mais surtout le fait assumé que l'objet produit puisse ne plus avoir de lien avec la quantique autre que la méthode qui y a mené rendent le projet singulier. Il s'agit d'éprouver la quantique, en tant que créateur, de mettre en scène une démarche, d'assumer une pleine réflexivité et non pas de produire un « art quantique ». 
Le projet "Trois petits chats » rend aussi compte d'un discours personnel assumé sur l'objet gadget, capable d'être à la fois canard et téléphone, homard et harmonica, etc. Ce projet comme celui d'Émile témoignent d'une mise à distance d'avec le discours scientifique et précisément se présentent comme une exploration plastique et esthétique de la physique quantique.

\section{CONCLUSION : LES SPÉCIFICITÉS DES OBJETS DE DESIGN}

La première conclusion que l'on peut tirer au regard de la description des projets est un étonnement. Le design opère remarquablement pour explorer tous les rapports possibles vis-à-vis du discours scientifique : certains objets affichent explicitement un rapport pédagogique, d'autres juxtaposent différents registres de discours (pédagogique, humoristique, fantastique), d'autres encore assument très explicitement une absence de contrôle du sens vis-à-vis du destinataire. Le design opère aussi pour explorer la diversité des formats des objets de communication de la science (ouvrage imprimé, production audiovisuelle, dispositif spatial, etc.). Cette conclusion peut également être tirée de la collaboration précédente, autour de l'atelier SupraDesign, qui traitait de la supraconductivité, et pour lequel les objets produits balayaient très largement le spectre des objets de médiation : jeu interactif, vidéo humoristique, cirque de marionnettes, sculpture artistique, objets futuristes, dessins ${ }^{32} \ldots$ C'est là un point essentiel qui caractérise l'apport du designer : le design permet d'explorer de façon continue quelque chose qui va du très rigoureux au très délirant. Ce n'est pas le cas, en général, des approches collaboratives avec d'autres disciplines. Par exemple avec des graphistes ou des web-designers, l'objet produit est « contraint » dans un format (numérique ou papier). Ici, l'absence de contrainte de format et de support a ouvert un large champ de possibles.

La seconde conclusion que nous pouvons tirer de cette expérience est que les objets de design sont, pour la plupart, des objets de médiation. Ces objets tissent un rapport non pas tant directement avec la science qu'avec la représentation formelle ou analogique de la science, ou alors avec les supports de communication de la science, ou avec la charge symbolique que comporte un territoire comme la science. Le rapport à l'infra-ordinaire et le décalage sont les deux ressorts principaux de cette construction. Le décalage a pour effet de produire des situations d'attention particulière. En effet, la surprise, l'étonnement, permet d'attirer l'attention et de créer les conditions d'une situation de communication. En outre, la construction d'un rapport tangible et visible à la quantique, sur fond de paysage infra-ordinaire, c'est-à-dire sans recours à des images sophistiquées, des personnages surnaturels, des technologies révolutionnaires, produit une corrélation puissante entre science et société. C'est cela qui permet aussi de faire la différence entre ces productions et les dispositifs de type art-science. L'artiste, lui aussi, produit un langage plastique et des formes. Mais ici le designer ne considère pas tant la forme pour elle-même, elle est un prétexte pour servir un propos. À ce titre, l'objet de design rend compte d'une commande et explicite d'une manière ou d'une autre le lien qu'il tisse avec cette commande.

Ce rapport de réflexivité à la commande participe certainement de la spécificité de l'objet de design, en tant qu'objet de médiation, par rapport à

32. Le site internet http://www.supradesign.fr présente le projet et les productions des élèves. 
l'artiste, à la médiation d'une institution muséale ou au scientifique, pour prendre trois types d'acteurs aux prises avec des situations de médiation de la science. En effet, si l'on se place dans la perspective d'une analyse des situations de conception des objets de médiation de la science et que l'on considère le design, non plus seulement du point de vue sémiotique, mais du point de vue économique et politique, on peut aussi le distinguer en tant qu'activité de production et de communication. Le designer opère dans un cadre qui lui offre une liberté " intermédiaire », et ce, à la différence du scientifique, du médiateur et de l'artiste. Pour le scientifique, c'est l'attachement à sa communauté scientifique qui structure la conception. Ce qui structure l'activité du médiateur est la légitimité dans le champ scientifique et l'attachement au cadre et au format de sa propre institution. Enfin, l'artiste semble soumis aux lois du marché de l'art et à la nécessité de développer des langages plastiques, poétiques, textuels. Le designer est en effet plus libre que le scientifique ou le médiateur, même s'ils sont les commanditaires du projet, car ces derniers n'engagent plus leur responsabilité auctoriale. Mais le designer est moins libre que l'artiste car il a un commanditaire (même dans notre cas : l'école et les institutions scientifiques partenaires). C'est probablement ce cadre sous contrainte «modérée » qui permet la grande diversité des productions, la grande palette des prises de distance vis-à-vis du discours scientifique et en même temps l'attachement et le détournement des formes traditionnelles de la médiation scientifique.

\section{Bibliographie}

Albe Virginie, Enseigner des controverses, Presses universitaires de Rennes, 2009

Barral Jacquie, Gilles Joël (dir.), Dessein, dessin, design, publications de l'université de SaintÉtienne, 2007

Baudrillard Jean, Le système des objets : la consommation des signes, Gallimard, Paris, 1968

Bauer Martin W., «Changement de paradigme de la communication scientifique. Un public critique pour la science commercialisée? ", Questions de communication, 21, 2012

Bordeaux Marie-Christine, "Une médiation paradoxale : "La danse, une histoire à ma façon" ", MEI - Médiation et information, 19, 2004

Callon Michel, "Quatre modèles pour décrire la dynamique de la science », in Madeleine Akrich, Callon Michel, Latour Bruno (dir.), Sociologie de la traduction. Textes fondateurs, Mines Paris, 2006

Callon Michel, «Plaidoyer en faveur du réchauffement des relations entre science et société. De l'importance des groupes concernés », L'Actualité chimique, 280-281, 2004

Cartellier Dominique, "La vulgarisation scientifique à l'heure de libre accessibilité des savoirs. Quelle place pour les médiateurs? », in Marie-Pier Luneau et Josée Vincent (dir.), La lecture: agents, pratiques, usages et discours, Volume 1, numéro 2, Groupe de recherche et d'étude sur le livre au Québec, 2010

Caune Jean, Pour une éthique de la médiation : Le sens des pratiques culturelles, Presses universitaires de Grenoble, 1999

Cheveigné Suzanne (de), "Enquête du CNRS sur les attentes du public vis-à-vis de la science ", CNRS Info, 381, 2000, citée par Christophe Bonneuil, "Les transformations des rapports entre sciences et société en France depuis la Seconde Guerre mondiale : un essai de synthèse », colloque Science, médias et société, 15-17 juin 2004, Lyon ENS-LSH

Collombat Isabelle, «Le discours imagé en vulgarisation scientifique : étude comparée du français et de l'anglais », metaphorik.de, 05/2003 
Davallon Jean, Flon Émilie, « Le média exposition », Culture et Musées, 2013

Davallon Jean, « Réflexions sur la notion de médiation muséale », L'Art contemporain et son exposition (1), L'Harmattan, Paris, 2002

Dubois Jean, Dictionnaire de Linguistique, Larousse, Paris, 1973

Guidot Raymond, Histoire du Design de 1940 à nos jours, Hazan, Paris, 2004

Jacobi Daniel, La communication scientifique : discours, figures et modèles, Presses universitaires de Grenoble, 1999

Jacobi Daniel, Bernard Schiele, (éd.), Vulgariser la science, Éditions Champ Vallon, Ceyzérieu, 1988

Jacobi Daniel, "Sémiotique du discours de vulgarisation scientifique », Semen [En ligne], 2 | 1985, mis en ligne le 21 août 2007, consulté le 30 septembre 2013. URL : http://semen.revues.org/4291

Jeanneret Yves, Penser la trivialité. Vol. 1 : La vie triviale des être culturels, Lavoisier, Paris, 2008

Jeanneret Yves, Écrire la science. Formes et enjeux de la vulgarisation, PUF, Paris, 1994

Jurdant Beaudoin, Les problèmes théoriques de la vulgarisation scientifique, Archives contemporaines, Paris, 2009

Keane Webb, "Semiotics and the social analysis of material things", Language \& Communication, 23(3-4), 2003

Krippendorff Klaus, The Semantic Turn: A New Foundation for Design, CRC Press, Boca Raton, 2005.

Le Marec, Joëlle (dir.), «Évolution des rapports entre sciences et société au musée », Culture et Musées, 10, 2007

Mortureux Marie-Françoise, « Paraphrase et métalangage dans le dialogue de vulgarisation ", Langue française, 53, 1982

Peraya Daniel, «Un regard critique sur les concepts de médiatisation et médiation. Nouvelles pratiques, nouvelle modélisation ", Les Enjeux de l'information et de la communication, 2008.

Perec George, L'infra-ordinaire, Éditions du Seuil, Paris, 1989

Pestre Dominique, (dir), «Sciences, Technologies, Savoirs en sociétés », Science \& devenir de l'homme, 57/58, numéro spécial, Éditions du Seuil, 2008

Quéré Louis, Miroirs équivoques, Aubier, Paris, 1982

Lévy-Leblond Jean-Marc, « Science, culture et public. Faux problèmes et vraies questions », Quaderni, 46, 2001-2002

Reboul-Touré Sandrine, "Le transgénique et le citoyen dans la presse écrite », Les Carnets $d u$ Cediscor [En ligne], 6 | 2000, mis en ligne le 30 novembre 2009, consulté le 3 août 2013. URL : http://cediscor.revues.org/342

Schiele Bernard, Le musée de sciences : montée du modèle communicationnel et recomposition du champ muséal, L'Harmattan, Paris, 2001

Shank Gary, "It's Logic in Practice, My Dear Watson: An Imaginary Memoir from Beyond the Grave", Forum: Qualitative Social Research, 2(1), Art. 9, 2001

Vandecasteele Éric, "les fictions du design (nains de jardin et pensée topologique) », in Barral, Jacquie Gilles Joël (dir.), Dessein, dessin, design, publications de l'université de Saint-Étienne, 2007

Verner Panton, «Qu'est-ce que le design », in Alexandra Midal (dir.), Design, L'anthologie. 1841-2007, Éditions de la Cité du design, Saint-Étienne, 2013

\section{CAMILLE JUTANT ET JULIEN BOBROFF}

